\title{
Everybody Knows Somebody
}

In contemplating this special focus on mental health and addictions, I reflected on numerous personal experiences with family, friends, colleagues and acquaintances. It was startling to realize the stunning number of people encountered over the course of my lifetime who have experienced or been affected by some form of mental illness and/or addiction. I suggest this as a reflection that everyone might benefit from periodically, if only to realize that mental health issues are not limited to the purview of the impoverished, the traumatized, the less educated or marginalized.
"It's a thief in the night

To come and grab you

It can creep up inside you

And consume you

A disease of the mind

It can control you

It's too close for comfort'

$\sim$ Disturbia, Rihanna

While many of us have what others might describe as quirky behaviors, are perhaps seen as a little (or a lot) eccentric, or are admittedly obsessive about certain things, it is likely that we are able to function in society without difficulty. On the other hand, we have likely all known people suffering from mental illness that interferes with day to day functioning, disrupts families, ends relationships, or in the extreme, leads to death by suicide. And while we may all have bouts of craving and secret or not so secret guilty pleasures, addictions have also taken a toll on the lives of individuals and their families, often equally devastating. Not my family, not my friends, not my people - not possible. Upon reflection of those you know or have known in your lifetime, in all likelihood there are many who have or continue to suffer from a mental illness, addiction, or both.

Over the course of the last two decades, much progress has been made in the approaches to the care of people with mental health and addiction challenges. Ken Kesey's (1963) One Flew Over the Cuckoo's Nest was an all too realistic portrayal of the horrific yet common fate of mentally ill persons that persisted in this country until the systematic closure of so named "insane asylums" in the latter half of the $20^{\text {th }}$ century. This move set the stage for stigma reduction, but not its elimination. Although approaches to the treatment of mental illness and addictions have also dramatically evolved over the last few decades, we have a lot more work to do in terms of advocacy and the provision of meaningful and effective support for these individuals. 
In this issue, our authors address an array of leadership issues related to the care of people with mental illness and addictions. Their discussions encompass the need for greater awareness and education of nurses, how patient partnerships are important and how active engagement can be enhanced through the use of technology, and perhaps most importantly, how nurse leaders can advocate for strategies that mitigate harm, reduce stigma and optimize outcomes. In the opening paper, the authors provide a broad overview of some of the key considerations for nurses and opportunities for leadership. Mildon discusses the role of nurses in recovery, maintaining safety and demonstrating accountability in the care of persons with mental illness while Cleverley addresses the critical role of nurses in maintaining continuity of care for persons with mental illness. The emerging role of technology in supporting and caring for individuals with mental health challenges is described by Strudwick, as she offers her perspectives on innovative approaches to care. As stigma remains a critical concern and challenge for those suffering from mental illness, Srivastava describes the efforts to mitigate its persistence in the developed world. And finally, Velji introduces the importance of establishing partnerships with clients seeking care for mental illness (Mildon et al. 2017).

Gehrs and colleagues (Gehrs et al. 2017) identify some of the current gaps in nursing leadership related to mental health and describe their efforts to support nurses' professional development and leadership capacity. Specifically, they describe a "unique scholarship, internship and mentorship model which aims to foster confidence, critical thinking and leadership competency development in the mental health and addictions context."

The important role of public health nursing in the promotion of mental health in infancy and early childhood is described and situated as a "health equity concern" by Marcellus and Shahram (2017). They highlight how "the burden of poor mental health disproportionately impacts people who are exposed to high-risk environments" (p. 44) and those lacking "the social, economic and environmental conditions required for good health." Further, they describe the evidence of early life stressors and trauma being consistently linked to mental as well as physical health problems in adulthood thus underscoring the importance of early intervention.

At the other end of the continuum, the growth of our aging and elderly population has resulted in an increased incidence of cognitive disorders, particularly dementia. Supporting the transitional care of these individuals has been challenging as they move between care sectors. Ritchie and colleagues (2017) describe an approach to maintain continuity of care for individuals with dementia using a simple technology solution. An electronic interprofessional care plan written from the patient's perspective has been demonstrated to effectively support care transitions from hospital to community. 
Ackerman, Virani and Billings (2017) share their views on the importance of assuming a consumer-oriented stance as they describe some of the new innovations in digital mental health. Addressing the issues of stigma and access to mental health services, they describe the provision of online self-help tools including a secure social networking tool, an artificial intelligence driven assistant that uses traditional cognitive therapy techniques, and a personalized mobile mindfulness meditation application. These tools exemplify the need to apply new thinking and techniques to take full advantage of our ubiquitously digitized world. Also using a novel approach, Maina and colleagues (2017) describe the process and outcomes of a community engagement and knowledge exchange forum designed to explore novel ways to prevent and respond to the substance abuse and addiction challenges in the Prince Albert region of Saskatchewan. Given the disproportionate impact of substance abuse and addictions, particularly among the large Indigenous population (39\%), the region has been challenged to address a number of population, community and social risks. Drawing upon participatory action research methods, this forum provided an opportunity to hear the voices of those that might otherwise be ignored. Similarly, focusing on patient partnership, Velji et al. (2017) emphasize the need for active engagement of those impacted by mental illness and addictions. In this piece, the authors describe how "patient as partner" has been actualized throughout their organization.

And finally, Chapman (2017) shares her very poignant, personal experience of a family member's tragic outcome of a drug addiction. Emphasizing that a shroud of stigma continues to impede essential care seeking for many of these individuals, she calls for the "humanization of people who use drugs." Moreover, she challenges us to put aside moralistic views, to be bold and identify opportunities for innovative, creative responses to the current overdose epidemic in this country.

The contributors to this special issue on mental health and addictions should give pause to our readership. Reflect on your views and potential biases, perhaps unease or even fear of those who are afflicted by mental illness and/or addiction. Think about the subtle or not so subtle stigma that endures despite highly visible and persistent campaigns designed to overcome it. Ask yourself, what can you do as a citizen and as a nurse leader to further advance effective approaches to the care and destigmatization of those with mental health and addiction challenges. Who among you has never known anyone? Everybody knows somebody. Do you know what you need to know to help and support the next somebody?

Lynn M. Nagle, RN, PhD, FAAN

Assistant Professor

Lawrence S. Bloomberg Faculty of Nursing

University of Toronto

Toronto, ON 


\section{References}

Ackerman, M.L., T. Virani and B. Billings. 2017. "Digital Mental Health - Innovations in ConsumerDriven Care." Canadian Journal of Nursing Leadership 30(3): 63-72. doi:10.12927/cjnl.2018.25384.

Chapman, L. 2017. "Lessons Learned After Losing my Brother to an Overdose. A Call to Action for Nurse Leaders.” Canadian Journal of Nursing Leadership 30(3): 73-79. doi:10.12927/cjnl.2018.25383.

Gehrs, M., G. Strudwick, S. Ling, E. Reisdorfer and K. Cleverley. 2017. "Addressing Gaps in Mental Health and Addictions Nursing Leadership: An Innovative Professional Development Initiative." Canadian Journal of Nursing Leadership 30(3): 23-42. doi:10.12927/cjnl.2018.25387.

Kesey, K. 1962. One Flew over the Cuckoo's Nest. New York, NY: Viking.

Maina, G., B. Mishak, A. de Padua, G. Strudwick, A. Docabo and H. Tahir. 2017. "Nurses Taking the Lead: A Community Engagement and Knowledge Exchange Forum on Substance Abuse and Addiction in Prince Albert, Saskatchewan." Canadian Journal of Nursing Leadership 30(3): 80-92. doi:10.12927/cjnl.2018.25382.

Marcellus, L. and S. Z. Shahram. 2017. "Starting at the Beginning: The Role of Public Health Nursing in Promoting Infant and Early Childhood Mental Health." Canadian Journal of Nursing Leadership 30(3): 43-53. doi:10.12927/cjnl.2018.25386.

Mildon, B., K. Cleverley, G. Strudwick, R. Srivastava and K. Velji. 2017. "Nursing Leadership: Making a Difference in Mental Health." Canadian Journal of Nursing Leadership 30(3): 8-22. doi:10.12927/ cjnl.2018.25388.

Ritchie, K., A. Duff-Woskosky and S. Kipping. 2017. "Mending the Cracks: A Case Study in Using Technology to Assist with Transitional Care for Persons with Dementia." Canadian Journal of Nursing Leadership 30(3): 54-62. doi:10.12927/cjnl.2018.25385.

Velji, K., C. Tyrell, M. Rice, A. Marshall and S. Riahi. 2017. “Patient Partnership: One Organization's Journey.” Canadian Journal of Nursing Leadership 30(3): 93-103. doi:10.12927/cjnl.2018.25381.

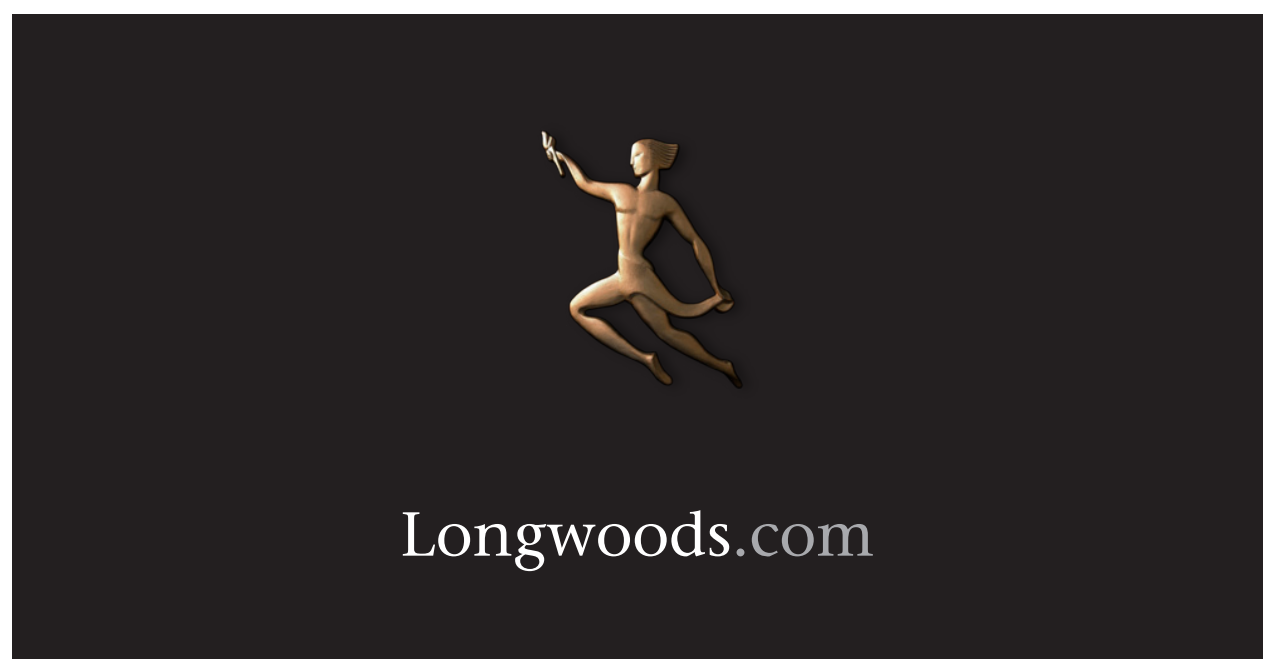

